\title{
GEOMETRIC EFFECTS ON ELECTRON CLOUD
}

\author{
L. Wang ${ }^{\#}$ and A. Chao, SLAC, CA 94025, USA \\ J. Wei, BNL, Upton, NY 11973, USA
}

\section{Abstract}

The development of an electron cloud in the vacuum chambers of high intensity positron and proton storage rings may limit the machine performances by inducing beam instabilities, beam emittance increase, beam loss, vacuum pressure increases and increased heat load on the vacuum chamber wall. The electron multipacting is a kind of geometric resonance phenomenon and thus is sensitive to the geometric parameters such as the aperture of the beam pipe, beam shape and beam bunch fill pattern, etc. This paper discusses the geometric effects on the electron cloud build-up in a beam chamber and examples are given for different beams and accelerators.

\section{INTRODUCTION}

Electron cloud in the beam pipe due to multipacting can cause transverse beam instabilities, beam loss, vacuum pressure rise and transverse beam emittance increase. Multipacting is induced by the beam itself because electrons gain energy from the beam. Effects of electron cloud have been observed in almost all the recent high intensity rings, such as LANL PSR, KEKB, PEPII, AGS, SPS [1]. Besides the secondary emission property of the material of the beam pipe, the geometric parameters, such as pipe aperture, longitudinal and transverse beam profile, bunch spacing, bunch current, etc., play an important role on the buildup of the electron cloud. This paper briefly summarizes the geometric effect to the bunch length in different accelerators.

\section{SHORT-BUNCH BEAM}

In accelerators like RHIC, LHC, SPS, and FNAL Main Injector, the bunch length ranges from about $0.1 \mathrm{~m}$ to $1 \mathrm{~m}$. Table 1 lists the main parameters for the three rings. An electron typically makes a few oscillations under the beam electric field during the bunch passage and the electron cloud in these machines is sensitive to the average bunch density. A transverse rms beam size of 2.4 $\mathrm{mm}$ is used in the calculations of this section.

Table 1: Main parameters of the three rings

\begin{tabular}{|l|l|l|l|}
\hline Variable & FNAL MI & RHIC & SPS \\
\hline Beam size $\sigma_{r}(\mathrm{~mm})$ & 5.0 & 2.4 & 2.2 \\
\hline Chamber radius $b(\mathrm{~mm})$ & $61.5 / 24.5$ & $35 \sim 60$ & $22 / 70$ \\
\hline Full Bunch length $L(\mathrm{~ns})$ & 9 & $5 \sim 35$ & $3 \sim 15$ \\
\hline Charge intensity $N\left(10^{10}\right)$ & $6 \sim 30$ & $10 \sim 20$ & $5 \sim 10$ \\
\hline Bunch spacing $t s p(\mathrm{~ns})$ & 19 & 108 & 25 \\
\hline
\end{tabular}

The electric field of a round Gaussian beam is

$$
E_{r}(r, t)=\frac{\lambda(t)}{4 \pi \varepsilon_{0}} \frac{2}{r}\left(1-e^{-r^{2} / 2 \sigma_{r}^{2}}\right)
$$

Where $r$ is the radial position, $\sigma_{r}$ is transverse $r m s$ beam size and $\lambda$ is beam line density which is given by the longitudinal beam profile, such as Gaussian beam,

$$
\lambda_{r}(t)=e N e^{-c^{2} t^{2} / 2 \sigma_{z}^{2}} /\left(\sqrt{2 \pi} \sigma_{z}\right)
$$

Here $N$ is the bunch charge number and $\sigma_{z}$ is the rms bunch length. The equation of an electron's motion under the beam's electric field is

$$
\ddot{r}(t)=\frac{2 N r_{e} c^{2}}{\sqrt{2 \pi} \sigma_{z}} \frac{2}{r}\left[1-e^{-r^{2} / 2 \sigma_{r}^{2}}\right] e^{-c^{2} t^{2} / 2 \sigma_{z}^{2}} .
$$

For the surviving electrons from the last bunch gap, the initial conditions of their motion can be assumed as $r(-\infty)=R$ and $\dot{r}(-\infty)=0$, then we get

$$
\dot{r}^{2}(t)=\frac{4 N r_{e} c^{2}}{\sqrt{2 \pi} \sigma_{z}} \int_{-\infty}^{t} d t \frac{1}{r}\left[1-e^{-r^{2} / 2 \sigma_{r}^{2}}\right] e^{-c^{2} t^{2} / 2 \sigma_{z}^{2}}
$$

Zero initial kinetic energy is a good assumption for these accelerators where the bunch spacing is long enough for all the electrons to drift to the surface. Therefore, the electrons which meet the following bunches are the secondaries from the surface which typically have a low energy of a few eVs. As a result, the energy gain here is independent of the secondary emission yield.

The energy gain (electron's energy after the passage of the whole bunch) (eq.4) is sensitive to $\sigma_{z}$ and $R$. Figure 1 shows the energy gain for different $\sigma_{z}$ and $R$ with $N=1 \times 10^{11}$ and $N=2 \times 10^{11}$. For a given bunch length, the energy gain as a function of $R$ has several peaks. The maximum peak is located at $R$ where an electron oscillates about half a period during the passage of the bunch as shown in Figure 2. Similarly, the second and third peaks appear when the electron oscillates through 1.5 and 2.5 periods. On the other hand, the valleys appear at $R$ when the electron oscillates integral multiple periods during the bunch passage. Due to the existence of the maximum energy gain, the observed electron's energy spectrum has a cutoff peak [2].

The bunch length plays an important role in the electron cloud build-up due to the sensitivity of electron energy gain to the bunch length (Eq.4). Figure 2 shows the peak energy gain and $R$ at the peak energy gain for various bunch charge and bunch length. Studies show the peak energy gain is linearly proportional to the average bunch line density with the scaling law as

$$
\Delta \hat{E}(e V)=150 \bar{\lambda}=42.31 e N / L
$$

where $\bar{\lambda}=\mathrm{Ne} / L$ is the beam line density in $C / s$ and $L$ is the full bunch length in second, $L=2 \sqrt{\pi} \sigma_{z} / \beta / c$ for a gaussian bunch. Eq. 5 is given for a $\sigma_{r}$ of $2.4 \mathrm{~mm}$. A small $\sigma_{r}$ gives a larger peak SEY. Beam size $\sigma_{r}$ is effective only at small $R$. The radial position with peak energy gain increases linearly with bunch line density:

\# email address: wanglf@slac.stanford.edu 


$$
\hat{R}=r_{\text {min }}(L)+k(L) \bar{\lambda}
$$

Both $r_{\min }$ and $k$ are constant parameters which are functions of bunch length only and both increase with bunch length. With a small beam pipe, it would be possible to find a set of parameters (bunch length and bunch intensity) where the energy gain is below the multipacting energy (e.g. 100eV) in the whole chamber region. The electron cloud can be mitigated this way. For example, there is no multipacting with a bunch length of $15 \mathrm{~ns}$, bunch charge intensity $2 \times 10^{11}$ and a beam pipe aperture of $25 \mathrm{~mm}$ (Fig. 1). It may be possible to apply this at the interaction region where strong electron cloud multipacting occurs. However, this idea may limit the choices of bunch length and intensity. A detailed study for certain realistic parameters would be advisable.

Electrons generated during the bunch passage due to beam ionization and beam loss at the chamber surface have different characteristics. The energy gain of these electrons depends on its birth time and position. They are born with a radial position $r_{0} \sim \sigma_{r}$ and $b$, respectively. Here $b$ is the radius of beam pipe. Figure 3 shows the energy gain of this type of electrons. Electrons produced by ionization can't cause multipacting due to their low energy gain. On the other hand, electrons generated at the beam pipe surface during the passage of the head of the bunch may receive enough energy for multipacting if the bunch length is long and the aperture of the beam pipe is small. When the surviving electrons meet the following bunches, they may receive energy from these bunches and multipacting occurs no matter what type of electrons they were at birth. Therefore, the overall effect of the electron's seed is not important for the electron build-up of the RHIC type of beam, where multipacting depends on multi-bunches.
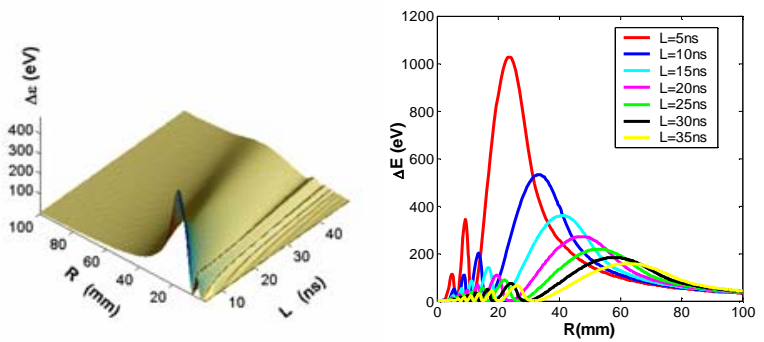

Figure 1: Energy gain for different radial positions and bunch lengths. Electrons are assumed initial to have zero energy at the initial position $R$. The bunch charge is $1 \times 10^{11}$ (left) and $2 \times 10^{11}$ (right).
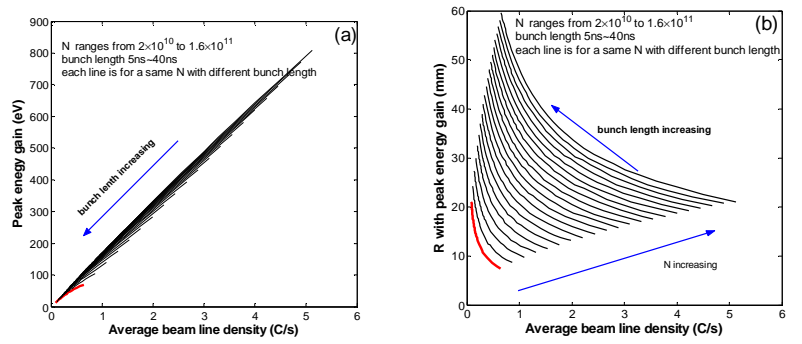

Figure 2: Average beam density effects on peak energy gain and its radial position. The bunch charge is $1 \times 10^{11}$.
When the electron cloud meets a beam with positive charge, the electrons are attracted by the beam's space charge force and therefore there is a large electron density around the bunch tail, which may cause instability and loss of beam particles at the tail.

Figure 4 shows the beam pinch effect with zero temperature for different bunch lengths. The electron density near the beam varies with time during the bunch passage. It has been normalized by the initial electron density to clearly see the pinch effect. Different from the peak energy gain [multipacting], there is a stronger pinch effect for a longer bunch length, such as with a $20 \mathrm{~ns}$ and $30 \mathrm{~ns}$ bunch. For a short bunch length, the strongest pinch happens at the bunch tail and it moves toward the bunch center when the bunch length increases. Figure 5 shows the electron cloud distribution due to the beam's pinch effect. The initial electron cloud distribution is from the CLOUDLAND simulation [3]. The high intensity of electron cloud near the beam due to the beam pinch effect can drive fast beam instability and cause beam loss at bunch tail.

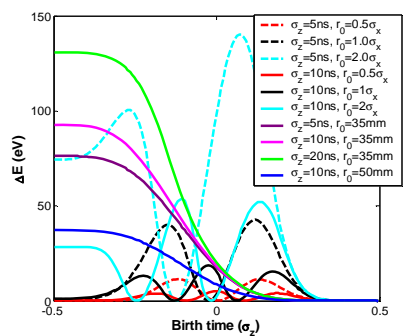

Figure 3: Energy gain of electrons generated by ionization and beam loss during the bunch passage. The bunch charge is $1 \times 10^{11}$. A negative birth time means bunch head.

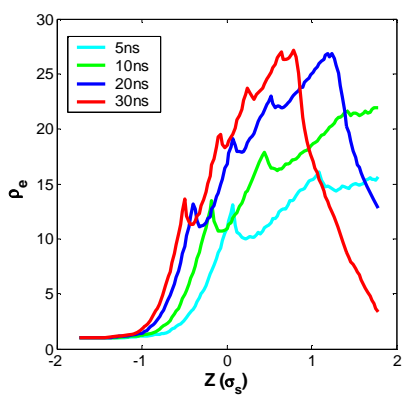

Figure 4: Pinch effect in RHIC for different bunch lengths. The horizontal-axis is the position normalized by bunch length and the vertical axis is the electron density inside the beam normalized by the initial electron density before the bunch arrival. The assumed charge per bunch is $8.79 \times 10^{10}$.
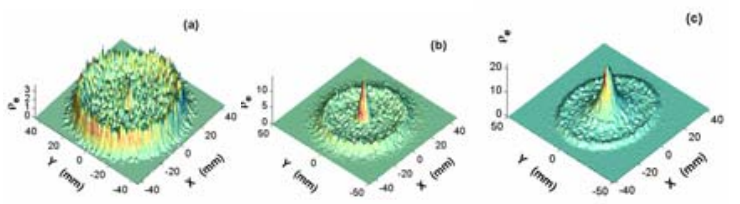

Figure 5: Electron cloud distributions during the bunch passage (beam's pinch effect) (a) $\mathrm{t}=-0.38 \sigma_{\mathrm{s}},(\mathrm{b}) \mathrm{t}=0$, (c) $\mathrm{t}=0.5 \sigma_{\mathrm{s}}$. All the electron densities are normalized by the initial electron density. 


\section{LONG BUNCH BEAM}

For a long bunch of a hundred meters, like the SNS and PSR beam, the beam's line density $\lambda(t)$ does not change much within one period of an electron oscillation, there is an adiabatic invariant for the electron motion. All electrons remaining inside the chamber before the approaching bunch (electrons surviving from the last bunch gap) can be trapped inside the beam during the bunch's passage and released at its end. These electrons receive less energy from the beam and therefore don't contribute to the electron multipacting, even though they may cause beam instability. The only multipacting electrons are those electrons which emit at the chamber's surface between the bunch's center and tail. They move straight to the opposite chamber wall and produce secondary electrons because the beam's profile has a negative derivative there [4]. The energy gain can be calculated by integrating along the electron' motion [4]

$$
\begin{aligned}
\Delta \mathrm{E}= & -\frac{1}{2} \beta c \sqrt{\frac{m e}{2 \pi \varepsilon_{0}}} \frac{\partial \lambda}{\partial z} \frac{1}{\sqrt{\lambda}}\left[a(2 \zeta-1) \arcsin \frac{1}{\sqrt{\zeta}}+a \sqrt{2 \ln \frac{b}{a}}+\right. \\
& \left.\sqrt{2} \zeta \int_{a}^{b} \frac{d r}{\sqrt{\ln (b / r)}}-\frac{1}{\sqrt{2}} \int_{a}^{b} \frac{1+2 \ln (r / a)}{\sqrt{\ln (b / r)}} d r\right]
\end{aligned}
$$

Where $a$ is the transverse beam size with a uniform distribution, $e$ and $m$ is the charge and mass of an electron, $\zeta=1+2 \ln (b / a)$. When $\lambda$ doesn't change much during one electron oscillation period, the energy gain can be approximately expressed as [5]

$$
\Delta E \approx-\pi m b^{2} \dot{\omega}_{e} / 2
$$

With

$$
\begin{gathered}
\omega_{e}=\left[e I /\left(2 \pi c \varepsilon_{0} \beta m\right) /\left(a^{2}+2 b^{2} / \pi\right)\right]^{1 / 2} \\
I=\lambda \beta c
\end{gathered}
$$

From Eqs. (8-10), the approximation of the energy gain is

$$
\Delta E \approx-\frac{1}{2} \beta c \sqrt{\frac{m e}{2 \pi \varepsilon_{0}}} \frac{\partial \lambda}{\partial z} \frac{1}{\sqrt{\lambda}} \frac{\pi b}{2 \sqrt{(a / b)^{2}+2 / \pi}}
$$

Eq. (7) and (11) give the same dependence on $\lambda$. In general, the beam size $a$ is a few times smaller than $b$, therefore the energy gain is approximately independent of $a$ and linearly proportional to $b$. Figure 6 shows the energy gain from Eq. (7) for various $\lambda, a$ and $b$. The Gaussian profile has the larger energy gain and the longest multipacting time and therefore the strongest electron cloud. The elliptical profile has the lowest energy gain and electron cloud density. The sinusoidal and parabolic profiles have a similar energy gain and electron cloud. The electron cloud with a beam profile generated by ORBIT simulation is similar to the electron cloud with a sinusoidal profile.

Although the energy gain is not sensitive to the transverse beam size $a$, the electron cloud density inside the beam increases (or decreases) with a smaller (or larger) $a$ [4] and therefore a stronger (or weaker) beam instability is expected. For a given beam, a larger (or smaller) beam pipe causes a larger (or smaller) energy gain. But it also reduces (or increases) the multipacting frequency due to the change in drift time. Figure 7 shows the electron cloud build up with different pipe apertures. The electron cloud is at a maximum with a pipe radius of $10 \mathrm{~cm}$. A strong multipacting occurs near the bunch tail due to the high energy gain. This has been called trailing edge multipacting [6]. There is no multipacting for a coasting beam where the electrons are accumulated by beam trapping.

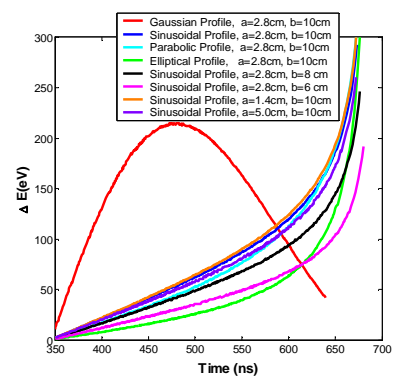

Figure 6: Energy gain of electrons emitted from a surface during the bunch tail. The bunch length is $700 \mathrm{~ns}$. The bunch charge is $2.05 \times 10^{14}$ (SNS beam).

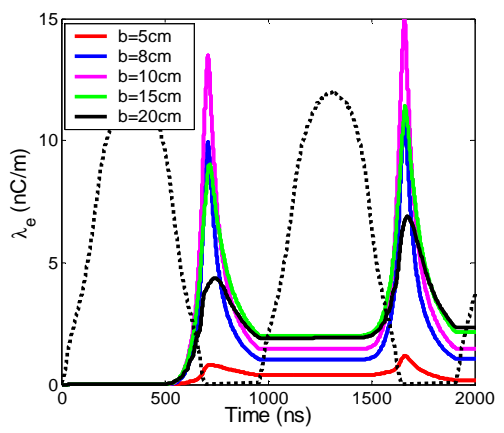

Figure 7: Effect of beam pipe aperture on electron cloud build-up. The bunch length is $700 \mathrm{~ns}$. The bunch charge is $2.05 \times 10^{14}$. The beam profile as shown by the back-dotted line is from an ORBIT simulation.

\section{ACKNOWLEDGEMENTS}

We thank Dr. S. Heifets for helpful discussions.

\section{REFERENCES}

[1] Mini-Workshop on Electron Cloud Simulation for Proton and Positron Beams, Proceedings ECLOUD'02, CERN-2002-001

[2] U. Iriso and W. Fischer, Phys. Rev. ST Accel. Beams 8, 113201 (2005)

[3] L. Wang, et.al., Phys. Rev. ST Accel. Beams v5, 124402 (2002)

[4] L. Wang, et. al., Phys. Rev. E 70, 036501(2004)

[5] M. Blaskiewicz, in Workshop on Instabilities of High Intensity Hadron Beams in Rings, AIP Conf. Proc. No. 496, 1991, p. 321.

[6] R. Macek, PSR Technical Note, PSR-00-10; also V. Danilov, et. al., Workshop on Instabilities of High Intensity Hadron Beams in Rings, AIP Conf. Proc. No. 496 (AIP, New York, 1999), p. 315. 